\title{
Emergence of Competition between Different Dissipative Structures for the Same Free Energy Source
}

\author{
Stuart Bartlett ${ }^{1,2}$, Seth Bullock ${ }^{2}$ \\ ${ }^{1}$ École Polytechnique Fédérale de Lausanne, CH-1015 Lausanne, Switzerland \\ ${ }^{2}$ University of Southampton, Southampton, SO17 1BJ, U.K. \\ stuart.bartlett@epfl.ch
}

\begin{abstract}
In this paper, we explore the emergence and direct interaction of two different types of dissipative structure in a single system: self-replicating chemical spot patterns and buoyancyinduced convection rolls. A new Lattice Boltzmann Model is developed, capable of simulating fluid flow, heat transport, and thermal chemical reactions, all within a simple, efficient framework. We report on a first set of simulations using this new model, wherein the Gray-Scott reaction diffusion system is embedded within a non isothermal fluid undergoing natural convection due to temperature gradients. The non-linear reaction which characterises the Gray-Scott system is given a temperature-dependent rate constant of the form of the Arrhenius equation. The enthalpy change (exothermic heat release or endothermic heat absorption) of the reaction can also be adjusted, allowing a direct coupling between the dynamics of the reaction and the thermal fluid flow.

The simulations show positive feedback effects when the reaction is exothermic, but an intriguing, competitive and unstable behaviour occurs when the reaction is sufficiently endothermic. In fact when convection plumes emerge and grow, the reaction diffusion spots immediately surround them, since they require a source of heat for the reaction to proceed. Then however, the proliferation of spot patterns dampens the local temperature, eventually eliminating the initial convection plume and reducing the ability of the spots to persist. This behaviour appears almost ecological, similar as it is, to competitive interactions between organisms competing for the same nutrient source.
\end{abstract}

\section{Introduction}

Spontaneous pattern formation is the primary exemplar of self-organisation in non-equilibrium systems, and remains a fascinating, enigmatic, and active field of research. Such phenomena has been observed and modelled in a wide range of systems including Dendritic Solidification (Ben-Jacob and Garik, 1990, Halsey, 2000, Vicsek, 1984), fluid convection (Pesch, 1996; Kadanoff, 2001), surfactant structures (Bachmann et al., 1992; Hanczyc and Szostak, 2004, Mayer et al., 1997; Ono, 2005), bacterial colony formation (BenJacob, 1997, 1993), and reaction-diffusion systems (Lee et al. 1994, Turing, 1952; Pearson, 1993, Gray and Scott. 1985, 1994, Mahara et al. 2008, Virgo, 2011), see also Gollub and Langer (1999); Prigogine (1978); Kondepudi and
Prigogine (2014). From an artificial life perspective, the dynamics of these (often very simple) systems are particularly poignant since they often exhibit properties analogous to those of living systems. We see behaviour such as selfreplication, precariousness (Virgo, 2011), adaptation and response to external conditions, even primitive forms of evolution.

We are compelled towards the conclusion that the phenomenological separation between non-living and living systems might be bridged by a continuum of simpler, socalled 'dissipative structures' (we assume that conscious, intelligent life is the most complex example of all). We can relate some of the emergent patterns listed above to the origin of life on Earth, in particular the well-known selfreplicating micelles, vesicles and droplets formed when surfactant molecules are dissolved in a polar solvent (Bachmann et al., 1992, Hanczyc and Szostak, 2004; Ono, 2005).

But we can also approach the problem on a more abstract level and pose the following question: Are the characteristic dynamics of life an inevitable, emergent property of nonequilibrium systems with a sufficient number of degrees of freedom and sufficient time? In this very general form, the answer is likely no, but it is still a worthy line of enquiry to try to delineate the types of systems, conditions, and timescales required for the emergence of life-like phenomena, in systems that are un-related to extant life. Thus in this work, we wished to explore whether non-living dissipative structures of different forms, might interact in ways reminiscent of biological systems.

The Gray-Scott reaction diffusion system (GSRDS) has been thoroughly investigated both theoretically (Gray and Scott, 1985, 1994; Mahara et al. 2008; Pearson, 1993 . Virgo 2011), and experimentally (Lee et al. 1994). One could even describe it as a descendant of the original ideas of morphogenetic pattern formation due to Turing (1952). The physical principles involved are simply the diffusion of two chemical species across a 2-dimensional domain, a nonlinear reaction between the two species, and the addition and removal of the two species to and from the domain. Within certain regions of the parameter space of this model, the bal- 
ance between supply of reactant, diffusion across concentration gradients, reaction into product, and removal of product, can produce a dizzying array of spontaneous patterns including self-replicating spots, stripes, waves, oscillations and spirals, among others. In this work we will be specifically interested in the self-replicating spot system, since we wish to explore the circumstances under which individual spots might compete not only with other spot patterns, but with completely different types of dissipative structure.

Alongside reaction diffusion phenomena, buoyancydriven convection of a single phase fluid stands as a characteristic archetype of emergent non-equilibrium pattern formation. Such systems are defined by the presence of a sustained temperature gradient and associated differential heating. This induces a buoyancy force due to density differences, setting in motion a flow of the fluid which can feed back on itself, producing rising plumes. Eventually a steady state (in the statistical sense for those systems that are turbulent) is reached in which a pattern of rolls or more complex structures facilitates the delivery of heat from the warmer to the colder boundary. The resulting heat flux is far greater than that which would occur by diffusion alone (see e.g., Ahlers et al., 2009; Bartlett and Bullock, 2014, Johnston and Doering, 2009, Malkus, 1954, Manneville, 2006, and references therein). The various flow patterns which emerge in these convective systems constitute another class of dissipative structure; they form in the presence of a free energy gradient, and act to reduce that gradient. It appears that they are a manifestation of the system trying to return to equilibrium. The formation of dissipative structures does however appear to hinder the approach to equilibrium in some cases (Awazu and Kaneko, 2004), but a full discussion of this observation is beyond the scope of this paper.

Given the robust, pattern-forming properties of GSRDS's and fluid convection, as discussed above, one could naturally ask whether these two phenomena might interact in the same system under certain conditions. It is this question which motivated the present work. The majority of GSRDS models assume that the two chemical species are dissolved within a motionless fluid. However the system can readily be modified such that the solvent is able to exhibit a sustained flow.

Reactive flow systems are of immense industrial importance and have hence attracted significant interest (see e.g., Andres and Cardoso, 2012, Berenstein and Beta, 2011; Kee et al. 2005, and references therein). However, the idea of embedding the GSRDS within a moving fluid has been explored to a lesser extent. Ayodele et al. (2013) analysed the effects of imposing a pre-defined flow field upon a GSRDS. They performed a linear stability analysis on this new, more complex system and defined the conditions under which the system was no longer robust to finite perturbations. It was demonstrated that a simple flow produces uniform translation of the reaction diffusion patterns (as one would expect from Galilean invariance), but that differential advec- tion can have a significant impact on the patterns formed by the chemical species concentrations.

While this paper achieved crucial milestones with regard to the modelling of reactive flow systems, it was only the chemical patterns which were an emergent result of the internal dynamics of the system. The flow fields were manually defined and imposed (they were boundary conditions). Similar systems have been modelled by other authors (Kreyssig and Dittrich, 2011). In the present work, we simulated systems in which both the chemical patterns and flow patterns emerged spontaneously. The primary achievement was the elucidation of conditions under which these two sets of phenomena directly interact with one another, in ways reminiscent of ecological phenomena.

In exploring this line of enquiry the principle difficulty was the construction of a simulation technique capable of representing the relevant physical processes, without being prohibitive in terms of computational power. Thus a new type of Lattice Boltzmann Model was derived and utilised (see Bartlett 2014, for a comprehensive description of the method and its development).

In this paper we first present the basic elements of the model, in the form required for answering the questions posed above. We then illustrate the most interesting phenomena so far observed in such systems, in particular the emergence of a competitive dynamic between the two different types of dissipative structure in the system. We then conclude and make suggestions for the further exploration of this area.

\section{The Chemical Lattice Boltzmann Model}

\section{Fluid dynamics}

The LBM is a kinetic-based computational fluid dynamics method that can be used to numerically model a vast array of physical systems including single-phase (Chen and Doolen, 1998), multi-phase (Shan and Chen, 1993) and multi-component fluids (Arcidiacono et al., 2007), magnetohydrodynamic systems (Chen et al., 1991), oil-watersurfactant systems (Chen et al., 2000; Nekovee et al., 2000) and reactive flow systems (Frouzakis, 2011), (see Chen and Doolen, 1998, for a comprehensive review of the LBM). It derives from the non-equilibrium Boltzmann equation:

$$
\frac{\partial f^{\prime}}{\partial t}+\mathbf{v} \nabla f^{\prime}=\Omega\left(f^{\prime}\right)
$$

which is the governing equation for the velocity distribution function $f^{\prime}(\mathbf{x}, \mathbf{v}, t)$. This represents the mass of particles per unit volume moving at velocity $\mathbf{v}$, measured over a small volume element centred on position $\mathbf{x}$ at time $t$. Changes in $f^{\prime}$ within this volume element occur through advection by the particle motion (at velocity $\mathbf{v}$ ), and through collisions between particles within the element, encapsulated in the collision term $\Omega\left(f^{\prime}\right)$. 
An appropriate discretisation and de-dimensionalisation leads to the evolution equation for the LBM on a discrete lattice:

$$
f_{i}\left(\mathbf{x}+\mathbf{e}_{i} \Delta t, t+\Delta t\right)-f_{i}(\mathbf{x}, t)=-\frac{1}{\tau_{\nu}}\left(f_{i}-f_{i}^{e q}\right)
$$

where $\Delta t$ is the time-step, $\tau_{\nu}$ is the relaxation time and $f_{i}^{e q}$ is the distribution of $f$ at equilibrium. Use of this equation assumes that the approach to equilibrium is characterised by a single time-scale. It can be shown that the appropriate equilibrium distributions are the following (Wolf-Gladrow, 2000),

$$
f_{i}^{e q}=\omega_{i} \rho\left[1+3 \frac{\mathbf{e}_{i} \cdot \mathbf{u}}{c^{2}}+\frac{9}{2} \frac{\left(\mathbf{e}_{i} \cdot \mathbf{u}\right)^{2}}{c^{4}}-\frac{3}{2} \frac{\mathbf{u}^{2}}{c^{2}}\right],
$$

where $\rho$ is the local fluid density, $\mathbf{u}$ is the local velocity, $c$ is the lattice spacing and $\mathbf{e}_{i}$ are the discrete velocity vectors. In this work, the 2-dimensional D2Q9 model will be used, which utilises a square lattice with 8 velocities and rest particles. For this velocity set, the weights $\omega_{i}$ are $\omega_{0}=4 / 9$, $\omega_{i}=1 / 9$ for $i=1,2,3,4$ and $\omega_{i}=1 / 36$ for $i=5,6,7,8$. The velocity vectors are thus:

$$
\begin{array}{r}
\mathbf{e}_{0}=(0,0) \\
\mathbf{e}_{1,3}, \mathbf{e}_{2,4}=( \pm c, 0),(0, \pm c) \\
\mathbf{e}_{5,6,7,8}=( \pm c, \pm c)
\end{array}
$$

To calculate macroscopic fluid variables, the appropriate moments of the distribution functions must be taken:

$$
\begin{array}{r}
\rho(\mathbf{x}, t)=\sum_{i} f_{i}(\mathbf{x}, t) \\
\rho(\mathbf{x}, t) \mathbf{u}(\mathbf{x}, t)=\sum_{i} \mathbf{e}_{i} f_{i}(\mathbf{x}, t)
\end{array}
$$

Using the Chapman-Enskog expansion, it can be shown that at the macro-level, a fluid obeying the above equations satisfies the Navier-Stokes equations with a kinematic viscosity given by:

$$
\nu=\frac{1}{3}\left(\tau_{\nu}-\frac{1}{2}\right) c^{2}
$$

The above equations are the main ingredients required to construct a LBM simulation for a single phase fluid. A large body of literature has demonstrated that this method can accurately simulate the behaviour of real fluids (see Chen and Doolen, 1998; Wolf-Gladrow, 2000, and references therein for further details concerning boundary conditions etc.).

\section{Passive Scalars}

As well as a single-phase fluid, the LBM can be modified to include extra components of various forms including internal energy (He et al., 1998, Peng et al., 2003, Shan, 1997) and passive scalars, such as chemical species (Ayodele et al.
2011, 2013; Arcidiacono et al., 2007). Let us first focus on the addition of the temperature field. All that is required is an extra distribution function:

$$
g_{i}\left(\mathbf{x}+\mathbf{e}_{i} \Delta t, t+\Delta t\right)-g_{i}(\mathbf{x}, t)=-\frac{1}{\tau_{c}}\left(g_{i}-g_{i}^{e q}\right)
$$

where $\tau_{c}$ is the internal energy relaxation time and $g_{i}^{e q}$ are the appropriate equilibrium distributions (He et al., 1998; Peng et al. 2003):

$$
\begin{array}{r}
g_{i=0}^{e q}=-\frac{2}{3} \rho \epsilon \frac{\mathbf{u}^{2}}{c^{2}} \\
g_{i=1,2,3,4}^{e q}=\frac{1}{9} \rho \epsilon\left[\frac{3}{2}+\frac{3}{2} \frac{\mathbf{e}_{i} \cdot \mathbf{u}}{c^{2}}+\frac{9}{2} \frac{\left(\mathbf{e}_{i} \cdot \mathbf{u}\right)^{2}}{c^{4}}-\frac{3}{2} \frac{\mathbf{u}^{2}}{c^{2}}\right] \\
g_{i=5,6,7,8}^{e q}=\frac{1}{36} \rho \epsilon\left[3+6 \frac{\mathbf{e}_{i} \cdot \mathbf{u}}{c^{2}}+\frac{9}{2} \frac{\left(\mathbf{e}_{i} \cdot \mathbf{u}\right)^{2}}{c^{4}}-\frac{3}{2} \frac{\mathbf{u}^{2}}{c^{2}}\right] .
\end{array}
$$

The internal energy density is represented by $\epsilon$ :

$$
\begin{array}{r}
\rho(\mathbf{x}, t) \epsilon(\mathbf{x}, t)=\rho(\mathbf{x}, t) R T(\mathbf{x}, t) \\
=\sum_{i} g_{i}(\mathbf{x}, t) .
\end{array}
$$

The diffusivity of this extra component (the thermal diffusivity) is given by

$$
\chi=\frac{2}{3}\left(\tau_{c}-\frac{1}{2}\right) c^{2} .
$$

To implement the effects of buoyancy, a forcing term must be added to the fluid evolution equation, which then reads,

$$
f_{i}\left(\mathbf{x}+\mathbf{e}_{i} \Delta t, t+\Delta t\right)-f_{i}(\mathbf{x}, t)=-\frac{1}{\tau_{\nu}}\left(f_{i}-f_{i}^{e q}\right)+F_{i},
$$

where

$$
F_{i}=\frac{\mathbf{G} \cdot\left(\mathbf{e}_{i}-\mathbf{u}\right)}{R T_{0}} f_{i}^{e q},
$$

$R$ is the molar gas constant, $T_{0}$ is the mean temperature and $\mathbf{G}=\beta g_{0}\left(T-T_{0}\right) \hat{j}$ is the vector representing the buoyant gravity force ( $\beta$ is the thermal expansion coefficient, $g_{0}$ is the acceleration of gravity and $T$ is the local temperature).

With these modifications and appropriate boundary conditions (e.g., fixed temperature, fixed flux or zero flux), it is possible to simulate buoyancy driven convective systems. Again, a large body of work (see e.g., He et al., 1998; Peng et al., 2003, Shan, 1997) has validated the accuracy of this model (up to certain values of the Rayleigh number, above which, modifications are required).

In a similar manner to internal energy, other passive scalars can be added to the model. They have analogous evolution equations:

$$
h_{i}^{\sigma}\left(\mathbf{x}+\mathbf{e}_{i} \Delta t, t+\Delta t\right)-h_{i}^{\sigma}(\mathbf{x}, t)=-\frac{1}{\tau_{\sigma}}\left(h_{i}^{\sigma}-h_{i}^{e q, \sigma}\right)
$$


where $\sigma$ denotes the particular component, and $\tau_{\sigma}$ is the relaxation time for that component. The equilibrium distributions take on the familiar form:

$$
\begin{array}{r}
h_{i=0}^{e q, \sigma}=-\frac{2}{3} \rho \psi_{\sigma} \frac{\mathbf{u}^{2}}{c^{2}} \\
h_{i=1,2,3,4}^{e q, \sigma}=\frac{1}{9} \rho \psi_{\sigma}\left[\frac{3}{2}+\frac{3}{2} \frac{\mathbf{e}_{i} \cdot \mathbf{u}}{c^{2}}+\frac{9}{2} \frac{\left(\mathbf{e}_{i} \cdot \mathbf{u}\right)^{2}}{c^{4}}-\frac{3}{2} \frac{\mathbf{u}^{2}}{c^{2}}\right] \\
h_{i=5,6,7,8}^{e q, \sigma}=\frac{1}{36} \rho \psi_{\sigma}\left[3+6 \frac{\mathbf{e}_{i} \cdot \mathbf{u}}{c^{2}}+\frac{9}{2} \frac{\left(\mathbf{e}_{i} \cdot \mathbf{u}\right)^{2}}{c^{4}}-\frac{3}{2} \frac{\mathbf{u}^{2}}{c^{2}}\right] .
\end{array}
$$

The diffusivity of each component is given by

$$
D_{\sigma}=\frac{2}{3}\left(\tau_{\sigma}-\frac{1}{2}\right) c^{2} .
$$

\section{Reactions}

Having described a thermal LBM which can simulate the advection and diffusion of passive scalars, let us now consider chemical reactions between a subset of those passive scalars. For convenience we will begin with an isothermal GSRDS. The equations of motion for the concentration fields of the two species are:

$$
\begin{gathered}
\frac{\partial \psi_{A}}{\partial t}=D_{A} \nabla^{2} \psi_{A}-\psi_{A} \psi_{B}^{2}+F\left(1-\psi_{A}\right) \\
\frac{\partial \psi_{B}}{\partial t}=D_{B} \nabla^{2} \psi_{B}+\psi_{A} \psi_{B}^{2}-(F+R) \psi_{B}
\end{gathered}
$$

with the following autocatalytic reaction occurring: $A+$ $2 B \rightarrow 3 B$. The first terms on the RHS's represent diffusion, the second terms correspond to the non-linear reaction. The final terms in the two equations represent the inward or outward fluxes of $A$ and $B$ from or to external reservoirs. The inward flux of $A$ is proportional to the feed rate $F$, and the local difference in concentration between $\psi_{A}$ and unity. Similarly, the removal of $B$ is proportional to $F+R$, and also to the local concentration difference between $\psi_{B}$ and 0 .

It is straightforward to implement these effects in the LBM. The distribution functions representing the mass of the two components evolve according to:

$$
\begin{array}{r}
h_{i}^{A}\left(\mathbf{x}+\mathbf{e}_{i} \Delta t, t+\Delta t\right)-h_{i}^{A}(\mathbf{x}, t)=-\frac{1}{\tau_{A}}\left(h_{i}^{A}-h_{i}^{e q, A}\right) \\
-\omega_{i} \psi_{A} \psi_{B}^{2}+F\left(1-\psi_{A}\right) \\
h_{i}^{B}\left(\mathbf{x}+\mathbf{e}_{i} \Delta t, t+\Delta t\right)-h_{i}^{B}(\mathbf{x}, t)=-\frac{1}{\tau_{B}}\left(h_{i}^{B}-h_{i}^{e q, B}\right) \\
+\omega_{i} \psi_{A} \psi_{B}^{2}-(F+R) \psi_{B} .
\end{array}
$$

Although the field of reactive LBMs is less mature than that of simple fluid LBMs, modelling of GSRDSs has already been carried out and validated by Ayodele et al.(2011). Further testing was carried out in Bartlett (2014).

\section{Enthalpy Changes}

We know from the work of (Ayodele et al. 2013) that uniform advection of GSRDS systems produces a simple translation of the chemical patterns. We also know that differential advection can influence the linear stability of those structures. However in this work we aimed to uncover the dynamics of thermally driven systems, where both the fluid convection and chemical reactions are critically dependent on local temperature, and also influence the local temperature. In order to simulate these non-isothermal reactive flows, we require a temperature dependence within the reaction rate constant (this rate constant is set to unity for the standard GSRDS). We thus make use of the Arrhenius equation:

$$
k(\mathbf{x}, t)=A e^{-E_{a} / T(\mathbf{x}, t)}
$$

where $k(\mathbf{x}, t)$ is the reaction rate at position $\mathbf{x}$ and time $t, A$ is a parameter known as the frequency factor, $E_{a}$ is the activation energy of the reaction, and $T$ is the local temperature. Finally we must add an extra term to the internal energy evolution equation to account for the uptake and release of heat due to reactions:

$$
\begin{array}{r}
g_{i}\left(\mathbf{x}+\mathbf{e}_{i} \Delta t, t+\Delta t\right)-g_{i}(\mathbf{x}, t)=-\frac{1}{\tau_{c}}\left(g_{i}-g_{i}^{e q}\right) \\
-\omega_{i} k(\mathbf{x}, t) \psi_{A} \psi_{B}^{2} \Delta H,
\end{array}
$$

where $\Delta H$ is the enthalpy change of the reaction (positive for endothermic reactions and vice versa). With this thermal reactive LBM, we are in a position to simulate all the necessary phenomena: buoyancy-driven fluid flow, advection and diffusion of heat, advection and diffusion of chemical species, and thermal reactions between species.

\section{Competition for Free Energy Access}

We now present an overview of the phenomena that can be exhibited by the above described model. For a more comprehensive exploration, please see Bartlett (2014).

First consider a system in which there is no uptake or release of heat during the reaction. Hence the presence of the chemical species does not influence the temperature field or fluid flow. The reaction rate is however influenced by the local temperature, and the chemical species are advected by the flow. The parameters used in these simulations are $E_{a}=1.7$ and $A=e^{E_{a} / T_{0}}$, where $T_{0}$ is the mean temperature. The evolution of the system is shown in Figure 1 .

We see that the convection of the fluid proceeds as normal (there is no feedback between the reaction and the fluid flow). The effects of the temperature dependent rate constant are also clearly visible. The temperature gradient between the warm lower boundary, cooler inner regions of the convection rolls, and cold upper boundary takes the chemical patterns from a steady state of pure $B$ at the bottom of the system, through stripes and worms to self-replicating spots, all the way to a region of pure $A$ near the top boundary. This 

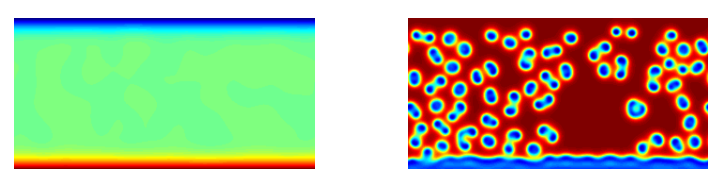

(a) $t=500$
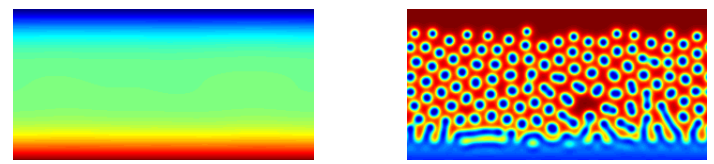

(b) $t=2200$
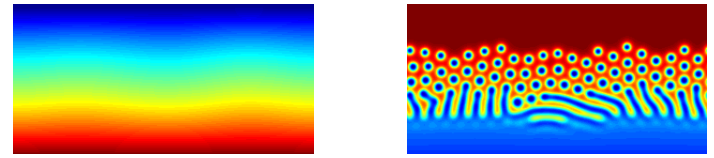

(c) $t=10600$
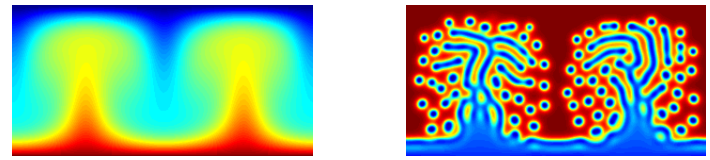

(d) $t=50000$

Figure 1: Temperature (left column) and chemical order parameter $\phi=\psi_{A}-\psi_{B}$ (right column) fields for a thermally neutral $(\Delta H=0)$, convective GSRDS at several different times through the simulation.

'phase boundary layer' is similar to the one visible in Figure 6.2 of (Bartlett, 2014), where it was caused instead by changes in the feed and removal rates, $F$ and $R$.

We can now explore the impact of allowing a heat release from the autocatalytic reaction between the two species. Figure 2 shows a strongly exothermic simulation with $\Delta H=-1 \times 10^{-3}$.

In this case we see a dominating positive feedback effect where the heat released from the reaction enhances the reaction rate further, releasing even more heat. This dynamic continues indefinitely and the entire system becomes swamped by the $B$ substance and its temperature continues to increase.

Finally, let us move to the endothermic case. Figure 3 shows a simulation in which $\Delta H=2 \times 10^{-3}$. We see that the convection pattern is still able to form (note that with an aspect ratio of 2, the fluid-only system sometimes forms two convection rolls, and sometimes four, as in Figure 1, therefore the difference in the number of rolls between these two simulations is not related to the chemical phenomena). What is also visible is that the reaction-diffusion 'phase boundary layer', seen in Figure 1, is present, but it seems to be slightly more confined to the lower end of the domain, and the proliferation of spot patterns is subdued in this endothermic sit-

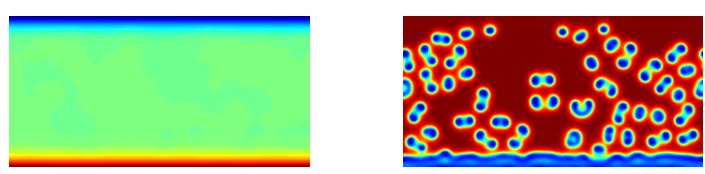

(a) $t=500$

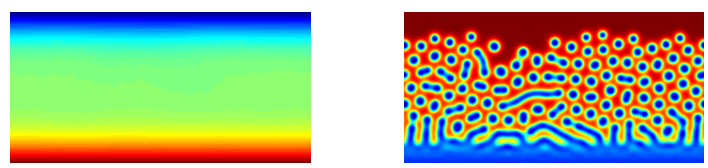

(b) $t=2200$

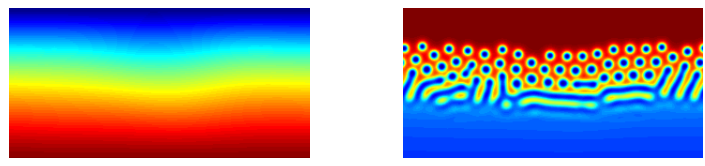

(c) $t=10600$

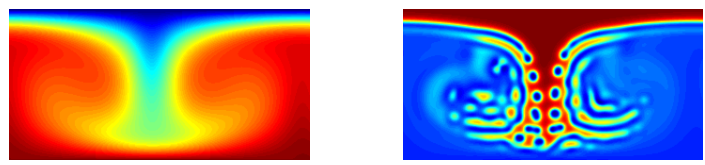

(d) $t=50000$

Figure 2: Temperature (left column) and chemical order parameter $\phi=\psi_{A}-\psi_{B}$ (right column) fields for an exothermic $\left(\Delta H=-1 \times 10^{-3}\right)$, convective GSRDS at several different times through the simulation.

uation. This is a result of the uptake of heat by the reaction, which forces the system to try to extract more heat from the lower boundary to compensate.

Now that we have observed an interaction between the two different types of dissipative structure (the reaction diffusion patterns affecting the heat flux, which itself is enhanced by the convection rolls), we can consider whether it is possible for the effects of one to completely diminish the other. We could for example, make the reaction even more endothermic.

When this is implemented, the reaction diffusion patterns end up confined to the lower boundary, the only place where the temperature can be maintained sufficiently high. If however, we reduce the activation energy of the reaction to $E_{a}=0.2$, and further increase the reaction enthalpy to $\Delta H=25 \times 10^{-3}$, a fascinating, oscillatory dynamic sets in. This is best viewed through the animation of the simulation: Bartlett (2015).

Initially, spots form across the domain, however they soon cool their environment so much that they can no longer persist. In fact the temperature begins to dip below even that of the cold upper boundary, and hence a small number of spots survive there. There is thus a large temperature gradient between the lower boundary, maintained at a high temperature, 

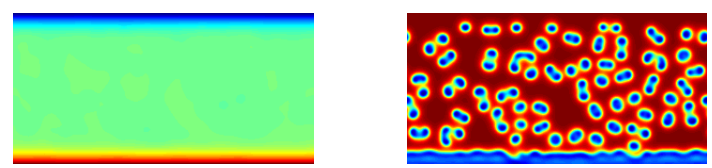

(a) $t=500$
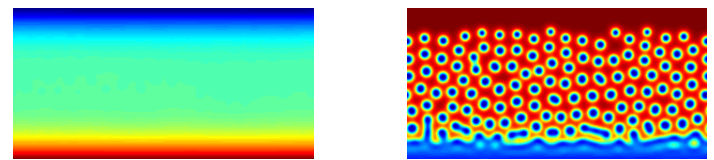

(b) $t=2200$
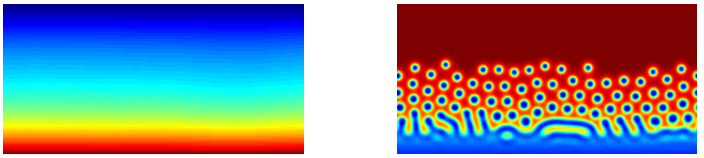

(c) $t=10600$
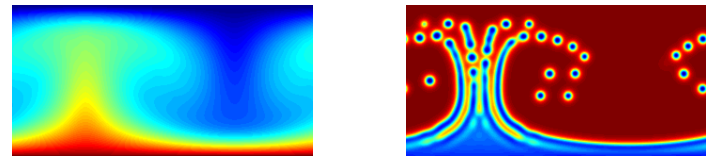

(d) $t=50000$

Figure 3: Temperature (left column) and chemical order parameter $\phi=\psi_{A}-\psi_{B}$ (right column) fields for an endothermic $\left(\Delta H=2 \times 10^{-3}\right)$, convective GSRDS at several different times through the simulation.

and the inner region of the domain. This gradient provides a strong driving force for the formation of convective plumes (the seeds of convection rolls), which sometimes burst into existence. However when this happens, there is a rapid proliferation of chemical activity, as the spot patterns appear to move to this new found heat source. The replication process removes heat from that locality, so much in fact that the initial convection plume becomes completely damped out. This competitive dynamic continues indefinitely, with the sporadic appearance of convection plumes, which are immediately leapt upon by 'clouds' of spot structures, the action of which leads to their own demise, and the demise of the plume.

In order to illustrate this interaction quantitatively, Figure 4 displays the time series of the vertical convective heat flux $Q_{y_{c}}=\overline{u_{y} T}$ (left axis and blue dashed line) and the spot pattern population $N_{R D S}$ (right axis and green solid line). It is difficult to numerically measure the proliferation of convection rolls, but the vertical convective heat flux at least illustrates changes in the degree of correlation between the temperature field and the vertical velocity field. It is this correlation which indicates the presence of spatially ordered heat movements. Tracking the population of reaction diffusion spots is somewhat more straightforward, given that they

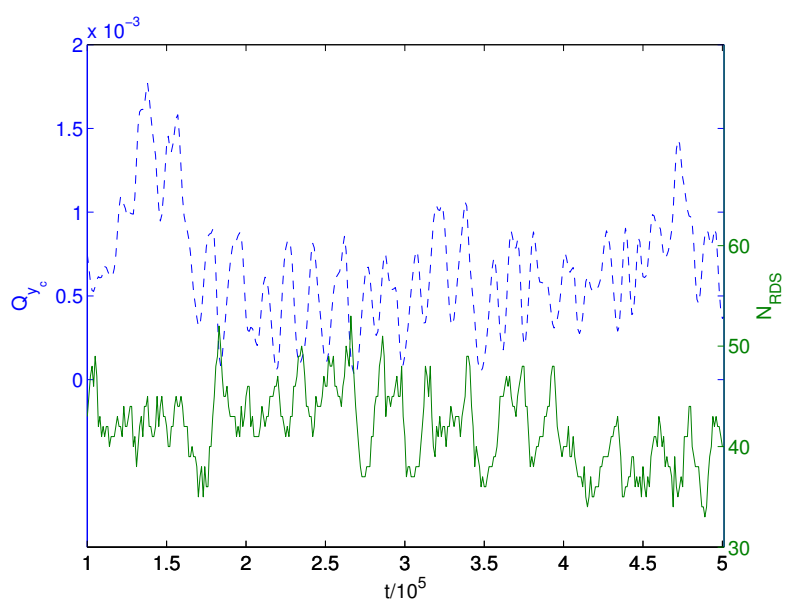

Figure 4: Population dynamics of the spot patterns within a thermal convective GSRDS. The left axis and blue dashed line corresponds to the vertical convective heat flux whereas the right axis and green solid line follows the number of individual spot patterns present in the system.

are discrete structures (apart from during their transient, division phase).

The two signals in Figure 4 are intermittent, but we see that there is a clear connection between them: they appear to be anti-correlated. The oscillations of both measures also seem to have a fairly consistent frequency. As expected from the animation of the simulation, most peaks in heat flux are followed by a peak in spot population. That peak then coincides with a reduction in heat flux. Indeed the increased growth of the spots causes a decline in heat flux. A thorough statistical analysis of this and other time series from the same ensemble is currently being carried out.

\section{Conclusions}

We have presented a new LBM capable of simulating an immense spectrum of physical phenomena. The full scope of the model can only be touched upon here. In this work, we have used it to explore the interactions between different types of dissipative structure, forming in a single system. The model is not a hybrid of different types, and does not require vast amounts of computational power. Its construction is also simple, intuitive, and physically-based. As shown by other authors previously, the isothermal chemical LBM can readily simulate simple chemical systems including the GSRDS.

What is novel about the work presented here is that we have relaxed the isothermal assumption and introduced a temperature dependent rate constant based on the Arrhenius equation. As far as we are aware, this is the first time that a thermal GSRDS has been simulated within a convective fluid. 
When the reaction does not release or absorb heat, the convection pattern forms as normal, and the chemical species exhibit a range of patterns across the spatial temperature gradient, due to the modulation of the rate constant with temperature. An exothermic scheme causes the reactive phase boundary to expand as it positively feeds back upon itself. With even more negative values of the enthalpy change, the positive feedback effect becomes so large that the system is overwhelmed by the single chemical species state.

Conversely, an endothermic reaction has a damping effect on both the proliferation of chemical structures and convection patterns. If the reaction is very endothermic then an oscillatory behaviour emerges, in which neither spot structures nor convection rolls can stably persist. Instead, any rising convective plume becomes rapidly surrounded by spot patterns, as the additional delivery of heat from the plume is taken up by the action of the endothermic spots.

What is remarkable about this behaviour is that it is reminiscent of an ecological scenario, in which two species, both competing for the same food source, deplete that source so quickly that it can no longer provide for either of them. Indeed a reduction in the enthalpy of reaction allows both spots and convection rolls to form. But when $\Delta H$ was sufficiently large, the spot patterns deplete a necessary component of their existence (a heat source) so fast that they destroy the conditions under which they can survive.

These results are suggestive of the possibility that ecological dynamics are more fundamental and older than biological phenomena. One can imagine other-worldly scenarios in which thermal or chemical gradients thrust elaborate patterns into existence, acting to reduce those gradients but being sustained by them. It seems likely that several such structures could coexist and interact. To take an example close to those in this paper, imagine if there were large gradients of chemical potential within the gases comprising Jupiter's red spot, and non-linear reactions between the relevant chemical species.

\section{Further Work}

The next chapter of this story should be to explore even more complex emergent ecologies in these reactive flow systems. Can these thermal spot patterns exhibit precariousness, the property of being able to emerge in a certain environment, and then go on to colonise regions that were previously inhospitable (Virgo, 2011)? An initial exploration of this question is presented in Bartlett (2014). Conditions under which the spots were shown to be precarious were not found. However, given the intimidating size and dimensionality of the parameter space, it is difficult to rule out any possibilities.

Might it be possible to observe strong hysteresis and some form of primitive evolution and adaptation of these structures? Ultimately, can this modelling paradigm help us uncover systems spanning the phenomenological gulf between non-living and living dissipative structures? Work to investigate these mysteries is currently in progress.

Another major direction to pursue is systems in which there is only one free energy source. Removing the feed and removal processes from the GSRDSs would achieve this, but then the characteristic patterns would not form. A materially closed system with only a temperature gradient across it was simulated in Bartlett (2014). There were two chemical species present with a simple linear reaction transforming one to the other. It was found that the presence of the chemical species enhanced the ability of the system to deliver heat from one boundary to the other. This enhancement was proportional to the total mass of chemical species dissolved in the solvent fluid. The next phase will be to employ more complex, nonlinear reaction schema such that individuated structures might appear.

\section{Acknowledgements}

This work was supported by an EPSRC Doctoral Training Centre grant (EP/G03690X/1).

\section{References}

Ahlers, G., Grossmann, S., and Lohse, D. (2009). Heat transfer and large scale dynamics in turbulent rayleigh-bénard convection. Rev. Mod. Phys., 81:503-537.

Andres, J. T. H. and Cardoso, S. S. S. (2012). Convection and reaction in a diffusive boundary layer in a porous medium: Nonlinear dynamics. Chaos: An Interdisciplinary Journal of Nonlinear Science, 22(3).

Arcidiacono, S., Karlin, I. V., Mantzaras, J., and Frouzakis, C. E. (2007). Lattice boltzmann model for the simulation of multicomponent mixtures. Phys. Rev. E, 76:046703.

Awazu, A. and Kaneko, K. (2004). Relaxation to equilibrium can be hindered by transient dissipative structures. Phys. Rev. Lett., 92:258302.

Ayodele, S., Raabe, D., and Varnik, F. (2013). Lattice boltzmann modeling of advection-diffusion-reaction equations: Pattern formation under uniform differential advection. Communications in Computational Physics, 13:741-756.

Ayodele, S. G., Varnik, F., and Raabe, D. (2011). Lattice boltzmann study of pattern formation in reaction-diffusion systems. Phys. Rev. E, 83:016702.

Bachmann, P. A., Luisi, P. L., and Lang, J. (1992). Autocatalytic self-replicating micelles as models for prebiotic structures. Nature.

Bartlett, S. (2014). Why is life? An assessment of the thermodynamic properties of dissipative, pattern-forming systems. $\mathrm{PhD}$ thesis, University of Southampton.

Bartlett, S. (2015). Reaction-diffusion-convection simulation with strongly endothermic reaction. https: / / www . youtube. $\mathrm{com} /$ watch? $\mathrm{v}=\mathrm{mI}$ fN6h-uRqE

Bartlett, S. and Bullock, S. (2014). Natural convection of a twodimensional boussinesq fluid does not maximize entropy production. Phys. Rev. E, 90:023014. 
Ben-Jacob, E. (1993). From snowflake formation to growth of bacterial colonies. Contemporary Physics, 34(5):247-273.

Ben-Jacob, E. (1997). From snowflake formation to growth of bacterial colonies ii: Cooperative formation of complex colonial patterns. Contemporary Physics, 38(3):205-241.

Ben-Jacob, E. and Garik, P. (1990). The formation of patterns in non-equilibrium growth. Nature, 343:523-530.

Berenstein, I. and Beta, C. (2011). Flow-induced control of chemical turbulence. The Journal of Chemical Physics, 135(16).

Chen, H., Boghosian, B. M., Coveney, P. V., and Nekovee, M. (2000). A ternary lattice boltzmann model for amphiphilic fluids. Proceedings of the Royal Society of London A: Mathematical, Physical and Engineering Sciences, 456(2000):2043-2057.

Chen, S., Chen, H., Martnez, D., and Matthaeus, W. (1991). Lattice boltzmann model for simulation of magnetohydrodynamics. Phys. Rev. Lett., 67:3776-3779.

Chen, S. and Doolen, G. D. (1998). Lattice boltzmann method for fluid flows. Annual Review of Fluid Mechanics, 30(1):329_ 364.

Frouzakis, C. (2011). Lattice boltzmann methods for reactive and other flows. In Echekki, T. and Mastorakos, E., editors, Turbulent Combustion Modeling, volume 95 of Fluid Mechanics and Its Applications, pages 461-486. Springer Netherlands.

Gollub, J. P. and Langer, J. S. (1999). Pattern formation in nonequilibrium physics. Rev. Mod. Phys., 71:S396-S403.

Gray, P. and Scott, S. (1994). Chemical Oscillations and Instabilities: Non-linear Chemical Kinetics. International Series of Monographs on Chemistry. Clarendon Press.

Gray, P. and Scott, S. K. (1985). Sustained oscillations and other exotic patterns of behavior in isothermal reactions. The Journal of Physical Chemistry, 89(1):22-32.

Halsey, T. C. (2000). Diffusion-limited aggregation: a model for pattern formation. Physics Today, 53(11):36-41.

Hanczyc, M. M. and Szostak, J. W. (2004). Replicating vesicles as models of primitive cell growth and division. Current opinion in chemical biology, 8(6):660-664.

He, X., Chen, S., and Doolen, G. D. (1998). A novel thermal model for the lattice boltzmann method in incompressible limit. Journal of Computational Physics, 146(1):282 - 300.

Johnston, H. and Doering, C. R. (2009). Comparison of turbulent thermal convection between conditions of constant temperature and constant flux. Phys. Rev. Lett., 102:064501.

Kadanoff, L. P. (2001). Turbulent heat flow: Structures and scaling. Physics Today, 54(8):34-39.

Kee, R., Coltrin, M., and Glarborg, P. (2005). Chemically Reacting Flow: Theory and Practice. Wiley.

Kondepudi, D. and Prigogine, I. (2014). Modern Thermodynamics: From Heat Engines to Dissipative Structures. Wiley.
Kreyssig, P. and Dittrich, P. (2011). Reaction flow artificial chemistries. In Advances in Artificial Life, ECAL 2011: Proceedings of the Eleventh European Conference on the Synthesis and Simulation of Living Systems, Paris, pages 431-437. MIT Press.

Lee, K.-J., McCormick, W. D., Pearson, J. E., and Swinney, H. L. (1994). Experimental observation of self-replicating spots in a reaction-diffusion system. Nature, 369(6477):215-218.

Mahara, H., Suzuki, K., Jahan, R. A., and Yamaguchi, T. (2008). Coexisting stable patterns in a reaction-diffusion system with reversible gray-scott dynamics. Phys. Rev. E, 78:066210.

Malkus, W. V. (1954). The heat transport and spectrum of thermal turbulence. Proceedings of the Royal Society of London. Series A. Mathematical and Physical Sciences, 225(1161):196212.

Manneville, P. (2006). Rayleigh-bénard convection: Thirty years of experimental, theoretical, and modeling work. In Mutabazi, I., Wesfreid, J. E., and Guyon, E., editors, Dynamics of Spatio-Temporal Cellular Structures, volume 207 of Springer Tracts in Modern Physics, pages 41-65. Springer New York.

Mayer, B., Köhler, G., and Rasmussen, S. (1997). Simulation and dynamics of entropy-driven, molecular self-assembly processes. Phys. Rev. E, 55:4489-4499.

Nekovee, M., Coveney, P. V., Chen, H., and Boghosian, B. M. (2000). Lattice-boltzmann model for interacting amphiphilic fluids. Phys. Rev. E, 62:8282-8294.

Ono, N. (2005). Computational studies on conditions of the emergence of autopoietic protocells. BioSystems, 81(3):223-233.

Pearson, J. E. (1993). Complex patterns in a simple system. Science, 261(5118):189-192.

Peng, Y., Shu, C., and Chew, Y. T. (2003). Simplified thermal lattice boltzmann model for incompressible thermal flows. Phys. Rev. E, 68:026701.

Pesch, W. (1996). Complex spatiotemporal convection patterns Chaos: An Interdisciplinary Journal of Nonlinear Science, 6(3):348-357.

Prigogine, I. (1978). Time, structure, and fluctuations. Science, 201(4358):777-785.

Shan, X. (1997). Simulation of rayleigh-bénard convection using a lattice boltzmann method. Phys. Rev. E, 55:2780-2788.

Shan, X. and Chen, H. (1993). Lattice boltzmann model for simulating flows with multiple phases and components. Phys. Rev. E, 47:1815-1819.

Turing, A. M. (1952). The chemical basis of morphogenesis. Philosophical Transactions of the Royal Society of London B: Biological Sciences, 237(641):37-72.

Vicsek, T. (1984). Pattern formation in diffusion-limited aggregation. Phys. Rev. Lett., 53:2281-2284.

Virgo, N. D. (2011). Thermodynamics and the structure of living systems. PhD thesis, University of Sussex.

Wolf-Gladrow, D. (2000). Lattice-Gas Cellular Automata and Lattice Boltzmann Models: An Introduction. Number no. 1725 in Lattice-gas Cellular Automata and Lattice Boltzmann Models: An Introduction. Springer. 